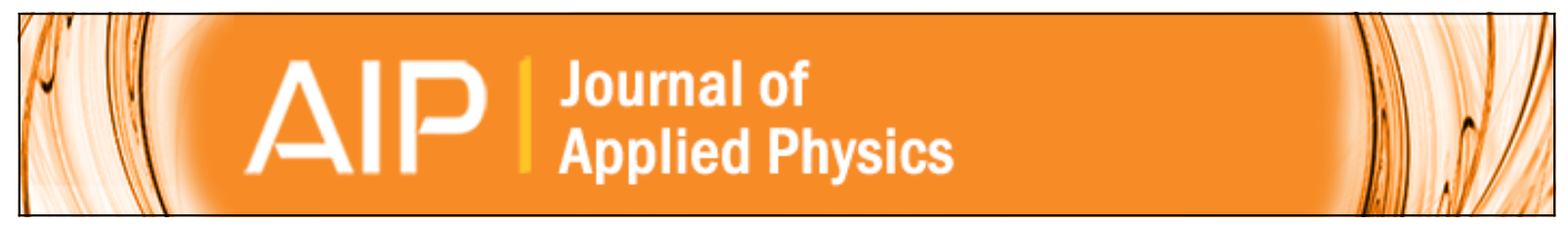

Modeling of organic light-emitting diodes with graded concentration in the emissive multilayer

A. Gusso, Dongge Ma, I. A. Hümmelgen, and M. G. E. da Luz

Citation: Journal of Applied Physics 95, 2056 (2004); doi: 10.1063/1.1640457

View online: http://dx.doi.org/10.1063/1.1640457

View Table of Contents: http://scitation.aip.org/content/aip/journal/jap/95/4?ver=pdfcov

Published by the AIP Publishing 


\title{
Modeling of organic light-emitting diodes with graded concentration in the emissive multilayer
}

\author{
A. Gusso \\ Instituto de Física Teórica (IFT)-Unesp, 01405-990 São Paulo SP, Brazil \\ Dongge Ma \\ State Key Laboratory of Polymer Physics and Chemistry, Changchun Institute of Applied Chemistry, \\ Chinese Academy of Sciences, Changchun 130022, People's Republic of China \\ I. A. Hümmelgen ${ }^{\text {a) }}$ and M. G. E. da Luz ${ }^{\text {b) }}$ \\ Departamento de Física, Universidade Federal do Paraná, C.P. 19044, 81531-990 Curitiba PR, Brazil
}

(Received 5 August 2003; accepted 18 November 2003)

\begin{abstract}
We model the electrical behavior of organic light-emitting diodes whose emissive multilayer is formed by blends of an electron transporting material, tris-(8-hydroxyquinoline) aluminum ( $\left.\mathrm{Alq}_{3}\right)$ and a hole transporting material, $N, N^{\prime}$-diphenyl- $N, N^{\prime}$-bis $\left(1,1^{\prime}\right.$-biphenyl)-4,4-diamine. The multilayer is composed of layers of different concentration. The $\mathrm{Alq}_{3}$ concentration gradually decreases from the cathode to the anode. We demonstrate that these graded devices have higher efficiency and operate at lower applied voltages than devices whose emissive layer is made of nominally homogeneous blends. Our results show an important advantage of graded devices, namely, the low values of the recombination rate distribution near the cathode and the anode, so that electrode quenching is expected to be significantly suppressed in these devices. (C) 2004 American Institute of Physics. [DOI: 10.1063/1.1640457]
\end{abstract}

\section{INTRODUCTION}

Since the reports on organic light-emitting diodes, ${ }^{1,2}$ much has been done to improve device efficiency and brightness, to reduce operation voltage, and to increase lifetime. A limitation commonly imposed by organic electroluminescent materials is that positive charge carriers have higher mobility than negative ones. ${ }^{3,4}$ Additionally, it is not easy to obtain $n$-doped organic semiconductors, ${ }^{5}$ and the energy barriers created by offsets between the metal cathode work function and the organic semiconductor lowest unoccupied molecular orbital (LUMO) inhibit negative charge injection. Under such conditions organic light-emitting devices tend to form electron-hole pairs predominantly in the cathode neighborhood. ${ }^{6-9}$ Light emission in the cathode neighborhood is suppressed due to quenching losses at the metal layer, being a crucial aspect for the overall device efficiency. ${ }^{10}$

Multilayer structures consisting of separate electron and hole transporting layers permit improvement in charge injection, transport and recombination., ${ }^{2,11}$ Blends of electron transport materials (ETMs) and hole transport materials (HTMs) were also used with the same intent. ${ }^{12-14}$ Along this line, graded emissive region devices achieved still better quantum efficiency, power efficiency and brightness than devices based on blends or bilayers. ${ }^{15}$ For the systems reported in Ref. 15, the emissive region is made of several layers, each one with a different relative concentration of ETM and HTM that compose the blend, respectively, tris-(8-

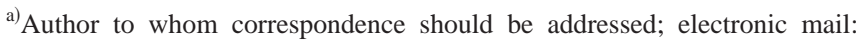
iah@ fisica.ufpr.br

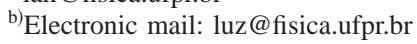

hydroxyquinoline) aluminum $\left(\mathrm{Alq}_{3}\right)$ and $N, N^{\prime}$-diphenyl$N, N^{\prime}$-bis $\left(1,1^{\prime}\right.$-biphenyl)-4,4-diamine (NPB). The concentration of NPB grows stepwise from the cathode to the anode, the opposite occurring with $\mathrm{Alq}_{3}$.

Our aim in this article is to present a simple model to explain the electrical behavior of the emissive region of graded multilayer organic light-emitting diodes like those of Ref. 15 and to investigate the conditions that improve their efficiency.

\section{THE MODEL}

\section{A. Single layer devices}

Based on a previous work, ${ }^{16}$ the authors in Ref. 17 discuss a model to describe bipolar current in single layer organic diodes, assuming that: (i) the mechanism of charge recombination is of Langevin type; (ii) the contribution of the diffusion current is small; (iii) the deep traps for both carriers can be neglected; and (iv) the mobilities of electrons and holes are field independent. The model leads to analytical expressions for the electric field and the charge densities that depend on the fraction of the total current density $J$ carried by the electrons at both the cathode and the anode. It allows a fairly good description of the device without knowing the exact physical process responsible for the charge injection at the interfaces, provided that the current fractions are known. Next, we outline some of the main ideas and results in Ref. 17, which will be necessary to the understanding of our model.

The total current flowing through the device is written as $J=J_{n}+J_{p}$, with $J_{n}=e \mu_{n} N F$ and $J_{p}=e \mu_{p} P F$. Here $F$ is the electric field, $P$ is the positive and $N$ the negative charge 
carrier densities, $e$ is the electron charge, and $\mu_{n}$ and $\mu_{p}$ are the electron and hole mobilities, respectively. $J_{n}, J_{p}, N, P$, and $F$ are all functions of the position $X$, that runs from 0 at the cathode to $L$ at the anode.

The device electrical behavior at steady state operation is governed by

$$
\begin{aligned}
& -\frac{1}{e} \frac{\partial J_{n}}{\partial X}=\frac{1}{e} \frac{\partial J_{p}}{\partial X}=\gamma N P, \\
& \frac{\partial F}{\partial X}=\frac{e}{\epsilon \epsilon_{0}}(N-P) .
\end{aligned}
$$

The first equation describes electron-hole recombination while the second is the Poisson equation. The Langevin recombination coefficient is given by $\gamma=e\left(\mu_{n}+\mu_{p}\right) / \epsilon \epsilon_{0}$ $=2 e \mu_{0} / \epsilon \epsilon_{0}$, were $\epsilon$ is the dielectric constant of the organic material, $\epsilon_{0}$ is the permittivity of vacuum, and $\mu_{0}$ is the average of the electron and hole mobilities.

It is convenient to introduce a set of dimensionless variables: $a=\left(\mu_{n} \mu_{p} \epsilon \epsilon_{0} F^{2}\right) /\left(2 \mu_{0} L J\right), B=J_{n} / J, C=J_{p} / J, x$ $=X / L(0 \leqslant x \leqslant 1)$, and $\nu_{n}=\mu_{n} / \mu_{0}, \nu_{p}=\mu_{p} / \mu_{0}\left(0 \leqslant \nu_{n}, \nu_{p}\right.$ $\leqslant 2$ ). This results in

$$
\begin{aligned}
& \frac{-\partial B}{\partial x}=\frac{\partial C}{\partial x}=\frac{B C}{a}, \\
& \frac{\partial a}{\partial x}=\left(2-\nu_{n}\right) B-\nu_{n} C, \\
& B+C=1 .
\end{aligned}
$$

$B(x)$ satisfies

$$
\frac{\partial B}{\partial x}=-K^{-1} B^{1-\nu_{n}}(1-B)^{\nu_{n}-1} .
$$

Defining $B_{c}=B(0)$ and $B_{a}=B(L)$ and using the following relation (with ${ }_{2} F_{1}$ the hypergeometric function): ${ }^{18}$

$$
\begin{aligned}
f(z, u)= & \int d z\left(\frac{1-z}{z}\right)^{1-u} \\
= & \frac{z^{u}}{u(u+1)}\left[(u+1){ }_{2} F_{1}(u, u, u+1 ; z)\right. \\
& \left.-u z_{2} F_{1}(u+1, u, u+2 ; z)\right],
\end{aligned}
$$

the constant $K$ is given by $K^{-1}=f\left(B_{a}, \nu_{n}\right)-f\left(B_{c}, \nu_{n}\right)$. For any $x, B(x)$ is obtained from the implicit equation

$$
f\left(B(x), \nu_{n}\right)-f\left(B_{c}, \nu_{n}\right)=-x K^{-1},
$$

with $a(x)$ given by

$$
a(x)=K B^{\nu_{n}}(1-B)^{2-\nu_{n}} .
$$

From $a(x)$ and $B(x)$ one obtains $F(x), J_{n}$, and $J_{p}$. The densities $N(x)$ and $P(x)$ follow from Eq. (1).

The expression relating the total current with the voltage drop across the whole single layer is obtained by integrating the electric field in the position variable and taking into account the built-in potential $V_{\mathrm{bi}}$ due to the different cathode and anode work functions. So, we have ${ }^{17}$

$$
\begin{aligned}
\frac{V-V_{\mathrm{bi}}}{J^{1 / 2}}= & \left(\frac{2 \mu_{0} L^{3}}{\epsilon \epsilon_{0} \mu_{e} \mu_{h}}\right)^{1 / 2} K^{3 / 2} \\
& \times \int_{B_{a}}^{B_{c}} d B B^{3 / 2 \nu_{n}-1}(1-B)^{2-3 / 2 \nu_{n}} .
\end{aligned}
$$

The dependence of $J$ on $\left(V-V_{\mathrm{bi}}\right)^{2} / L^{3}$ is typical of space charge limited currents.

In Ref. 17 it is discussed how the device radiance is related to $B_{c}-B_{a}$. In principle, the boundary conditions $B_{c}$ and $B_{a}$ could be obtained from such experimental measurements.

\section{B. Multilayer devices}

To extend the model described above to multilayer devices, we need to apply for each single layer the same basic equations of Sec. II A and introduce adequate matching conditions at the interfaces. In principle, quantities like the electric field or the electron density at the internal interfaces separating the layers could be infered from a detailed microscopic description of the injection mechanisms across such interfaces, but this is difficult to do. However, for graded devices such boundary conditions can be determined in a simple way, as we discuss next. A graded device has a multilayered structure where the concentration of the two materials has a different value in each layer. From the gradual variation of the concentrations we expect that the LUMO and highest occupied molecular orbital (HOMO) do not change considerably from layer to layer. The charge transport occurs via electrons that are injected to LUMO states and holes injected to HOMO states. If the LUMO and HOMO do not vary drastically along the device, we do not expect a strong accumulation of interfacial charge as observed when a gap exists between the LUMO or HOMO of different layers, ${ }^{19}$ which would result in abrupt changes in the electric field at the interfaces. ${ }^{20,21}$ Furthermore, if the van der Waals coupling between the molecules of the two materials is weak, their respective molecular orbitals remain basically unchanged. So, the electrons hop through the LUMO of one material and the holes through the HOMO of the other material until they recombine. In this case there is no heterojunction inside the recombination zone. These are then the plausible assumptions we make in our model. Finally, the parameter values that can change in the different layers are the electron and hole mobilities and the dielectric constants.

From the previous considerations, we require continuity of both the fraction of the current carried out by electrons, $B$, and the electric displacement vector $\mathbf{D}$ across any interface. The first is just a consequence of the steady state condition. The second results from neglecting any interfacial charge accumulation.

For simplicity, let us first discuss the graded bilayer case shown in Fig. 1. In terms of the electric field (to be more accurate, the component normal to the interface) and the dielectric constant, we have $\epsilon^{(1)} F_{12}=\epsilon^{(2)} F_{21}$, which will play the role of a matching condition. The subscript $i j$ means that the quantity is considered within the layer $i$ immediately before the interface between the layers $i$ and $j$. We have to solve the system of Eqs. (2) and (3) in both layers and need the quantities $B_{a}, B_{12}, B_{21}$, and $B_{c}$. In principle, $B_{c}$ is 


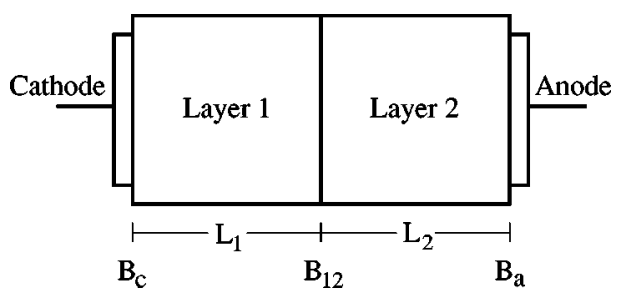

FIG. 1. Schematic representation of a bilayer device.

known at the interface with the cathode and $B_{a}$ at the interface with the anode (Fig. 1). Since the injection current is continuous, $B_{12}=B_{21}$. From Eq. (6), $\epsilon^{(1)} F_{12}=\epsilon^{(2)} F_{21}$ implies

$$
\frac{L_{1} B_{12}^{\nu_{e}^{(1)}}\left(1-B_{12}\right)^{2-\nu_{e}^{(1)}}}{\mu_{0}^{(1)} \nu_{e}^{(1)}\left(2-\nu_{e}^{(1)}\right)} K_{1}=\frac{L_{2} B_{12}^{\nu_{e}^{(2)}}\left(1-B_{12}\right)^{2-\nu_{e}^{(2)}}}{\mu_{0}^{(2)} \nu_{e}^{(2)}\left(2-\nu_{e}^{(2)}\right)} K_{2},
$$

where

$$
\begin{aligned}
K_{1} & =\int_{B_{12}}^{B_{c}} d B B^{\nu_{e}^{(1)}-1}(1-B)^{1-\nu_{e}^{(1)},} \\
K_{2} & =\int_{B_{a}}^{B_{12}} d B B^{\nu_{e}^{(2)}-1}(1-B)^{1-\nu_{e}^{(2)}} .
\end{aligned}
$$

The problem is then solved by substituting $B_{a}$ and $B_{c}$ by $B_{12}$ [obtained from Eq. (8)] into the corresponding integrals of Sec. II A.

For a multilayer device (Fig. 2) we repeat the above procedure for every pair of layers. For $N$ layers the device has $N-1$ internal interfaces and we have to solve $N-1$ equations like Eq. (8), simultaneously. The current-voltage characteristics are given by $\left(B_{01}=B_{c}\right.$ and $\left.B_{N N+1}=B_{a}\right)$

$$
\begin{aligned}
\frac{V}{J^{1 / 2}}= & \sum_{i=1}^{N}\left(\frac{2 \mu_{0}^{(i)} L_{i}^{3}}{\epsilon^{(i)} \epsilon_{0} \mu_{e}^{(i)} \mu_{h}^{(i)}}\right)^{1 / 2} K_{i}^{3 / 2} \\
& \times \int_{B_{i-1 i}}^{B_{i i+1}} d B B^{3 / 2 \nu_{e}^{(i)}-1}(1-B)^{2-3 / 2 \nu_{e}^{(i)}} .
\end{aligned}
$$

The above integral can be solved analytically. ${ }^{18}$

\section{Pure HTM and ETM layers}

The limits of integration of all integrals in the previous section are the values of the $B \mathrm{~s}$ at the interfaces. If one of such limits is either close to 1 (and $\nu_{n} \sim 2$ ) or close to 0 (and $\nu_{n} \sim 0$ ), then the resulting expressions become very sensitive to the system parameters (see Ref. 17 for more detail). The

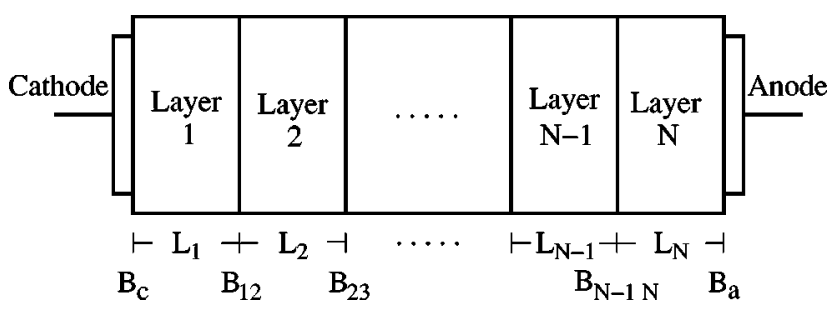

FIG. 2. Schematic representation of a multilayer device. first (second) condition takes place when in the first (last) layer $B_{c} \sim 1 \quad\left(B_{a} \sim 0\right)$ and the electron (hole) mobility exceeds the hole (electron) mobility by more than 2 orders of magnitude.

For graded systems there is a variation in the concentration, $r=[\mathrm{HTM}] /([\mathrm{ETM}]+[\mathrm{HTM}]),{ }^{22}$ ranging from smaller values (for the layers close to the cathode) to larger values (for the layers close to the anode). If for any layer in the multilayer device $r$ is not too close to 1 or 0 , then we can apply our model without any restriction. In some real applications,${ }^{15}$ however, the layers next to the cathode and to the anode may be composed of pure ETM and HTM, respectively. At such outmost layers we expect the values of $B_{c}$ and $B_{a}$ to be very close to 1 and 0 . Furthermore, $\nu_{n} \sim 2$ in the region where $B_{c}$ is only slightly smaller than 1 and $\nu_{n} \sim 0$ where $B_{a}$ is slightly above 0 . On the other hand, the remaining $N-2$ internal layers are made of a blend of HTM and ETM and consequently the values of $\mu_{n}$ and $\mu_{p}$ are typically of the same order of magnitude.

One of the purposes of the present model is to compare different graded devices, searching for the concentration profiles that optimizes the device performance under the reasonable assumption that $B_{c}$ and $B_{a}$ do not change appreciably around such a condition. The role of the first and last layers is mainly linked to carriers injection, while the power consumption and light emission will be determined essentially by the properties of the graded region. So, we can overcome the technical problem mentioned above by assuming plausible values for $B$ at the interfaces between the first and the second layers and between the $(N-1)$ th and $N$ th layers, thus omitting from the calculations the two outmost layers. An appropriate choice for these quantities can be made by observing that the electron-hole recombination should occur almost exclusively in the graded region. As a consequence, in the layer attached to the cathode the injected electrons have a small recombination rate and $B_{12}$ will be only slightly smaller than $B_{c}$. The same applies to the holes injected in the $N$ th layer, and consequently, $B_{N-1 N}$ is only slightly larger than $B_{a}$. We will take $B_{12}=0.98$ and $B_{N-1 N}=0.02$ in our simulations in Sec. III. We observe that to assume the $B \mathrm{~s}$ in the range $0.97<B_{12}<0.99$ and $0.01<B_{N-1 N}<0.03$ does not change qualitatively our results and conclusions.

\section{RESULTS}

Here, we will concentrate on the investigation of the concentration profiles that minimizes the $V / J^{1 / 2}$ relation, looking for the graded parameters which lead to the best device performance.

\section{A. The mobilities in a mixed single layer}

The first step is to know how the effective mobilities $\mu_{n}$ and $\mu_{p}$ depend on $r$. Results in the literature for some materials and mixing methods ${ }^{23}$ indicate that $\mu \propto r^{-n}(0.8 \leq n$ $\$ 2$ ). So, to cover a reasonable range of possibilities we analyze the following case that can be representative for materials of interest

$$
\mu_{n}(r)=a(b+r)^{-n},
$$


TABLE I. Concentration profiles of $\mathrm{Alq}_{3}$ and NPB that optimize the performance of some devices.

\begin{tabular}{|c|c|c|c|c|c|}
\hline$n$ & $n^{\prime}$ & {$\left[\mathrm{Alq}_{3}\right]:[\mathrm{NPB}]$} & $\begin{array}{c}V / J^{1 / 2} \\
\operatorname{grad} .\{\text { mix.\} }\end{array}$ & $r_{\min }$ & $\Delta_{P}$ \\
\hline 1 & 1 & $10: 1,6: 1,1: 1,1: 6,1: 10$ & $22.8\{26.3\}$ & 0.50 & $13 \%$ \\
\hline 2 & 2 & $2: 1,2: 1,1: 1,1: 2,1: 2$ & $18.0\{19.7\}$ & 0.49 & $09 \%$ \\
\hline $3 / 2$ & $3 / 2$ & $3: 1,2: 1,1: 1,1: 2,1: 3$ & $20.3\{22.5\}$ & 0.50 & $10 \%$ \\
\hline $3 / 2$ & 2 & $3: 1,2: 1,1: 1,1: 2,1: 3$ & $19.3\{21.0\}$ & 0.47 & $08 \%$ \\
\hline 1 & 2 & $7: 2,5: 2,3: 2,2: 3,1: 2$ & $20.9\{23.1\}$ & 0.44 & $10 \%$ \\
\hline
\end{tabular}

$$
\mu_{p}(r)=a^{\prime}\left(b^{\prime}+1-r\right)^{-n^{\prime}},
$$

where $n$ and $n^{\prime}$ can assume the values $1,3 / 2$, and 3 . The coefficients $a, a^{\prime}, b$, and $b^{\prime}$ are determined from the mobilities for pure ETM and HTM, requiring that $\mu_{n}(0)=\mu_{n}$ in pure ETM and $\mu_{n}(1)=\mu_{n}$ in pure $\mathrm{HTM} ; \mu_{p}(0)=\mu_{p}$ in pure ETM and $\mu_{p}(1)=\mu_{p}$ in pure HTM. Experimental data show that a blend of $\mathrm{Alq}_{3}$ and poly-vinyl carbazole, for example, follows the above functional relation. ${ }^{24}$

\section{B. Parameters values}

In order to compare our results with the experimental data in Ref. 15, we use typical parameters values for $\mathrm{Alq}_{3}$ and NPB as our ETM and HTM, respectively. For $\mathrm{Alq}_{3}$ both the electron and the hole mobilities were measured. ${ }^{25}$ For NPB only the hole mobility is known. ${ }^{26}$ For $\mu_{n}$ in $\mathrm{Alq}_{3}$ and $\mu_{p}$ in NPB the dependence on the electric field is quite weak. The main hole transport material is the NPB, with $\mu_{p}(\mathrm{NPB})$ being 3 orders of magnitude larger than $\mu_{p}\left(\mathrm{Alq}_{3}\right)$. For the electric fields calculated inside the devices in this work, $\mu_{p}$ in $\mathrm{Alq}_{3}$ would not vary significantly due to its field dependence. ${ }^{25}$ So, it is not a severe approximation to assume here that $\mu_{p}(\mathrm{NPB})$ is independent of $F$. We use the following values for the mobilities (in $\left.\mathrm{cm}^{2} / \mathrm{V} \mathrm{s}\right):^{25,26}$

$$
\begin{array}{ll}
\mu_{n}\left(\mathrm{Alq}_{3}\right)=1 \times 10^{-5}, & \mu_{p}\left(\mathrm{Alq}_{3}\right)=1 \times 10^{-7}, \\
\mu_{n}(\mathrm{NPB})=1 \times 10^{-7}, & \mu_{p}(\mathrm{NPB})=2 \times 10^{-4} .
\end{array}
$$

In the absence of any measurement for $\mu_{n}(\mathrm{NPB})$, we take its value based solely on the fact that in many organic materials the electron mobility is about 2 orders of magnitude smaller than the hole mobility.

We assume $\epsilon=3.5$, the value determined for pure NPB and pure $\mathrm{Alq}_{3} \cdot{ }^{19}$ Finally, we consider in our analysis that the total thickness of the graded region is always $50 \mathrm{~nm}$ as in Ref. 15, with the individual layers having the same size.

\section{Simulations}

Here we discuss the results for $N=7$ (Fig. 2), with the five inner layers forming the graded region as in the real devices of Ref. 15. For fixed $n$ and $n^{\prime}$ and a given concentration profile (represented in the form $\left[\mathrm{Alq}_{3}\right]:[\mathrm{NPB}]$ for each inner layer), we obtain the $r$ s and the $\mu$ s for all layers in the graded region, and from Eq. (10) we calculate $V / J^{1 / 2}$. The optimization of the $J \times V$ characteristic consists in finding the concentration profile that leads to a $V / J^{1 / 2}$ minimum. For different $n$ and $n^{\prime}$ we show in Table I the corresponding optimum profiles and the values of $V / J^{1 / 2}$. In Table II we
TABLE II. The coefficients in Eq. (11) for $n, n^{\prime}=1,3 / 2,2$ with the mobilities for pure $\mathrm{Alq}_{3}$ and NPB given by Eq. (12). The $a$ s are in $\mathrm{cm}^{2} / \mathrm{V} \mathrm{s}$ and the $b \mathrm{~s}$ are adimensional quantities.

\begin{tabular}{cccccc}
\hline \hline$n$ & $n^{\prime}$ & $a \times 10^{7}$ & $a^{\prime} \times 10^{7}$ & $b \times 10^{2}$ & $b^{\prime} \times 10^{2}$ \\
\hline 1 & 1 & 1.0101 & 1.0005 & 1.010 & 0.050 \\
$3 / 2$ & $3 / 2$ & 1.0739 & 1.0095 & 4.868 & 0.634 \\
2 & 2 & 1.2345 & 1.0463 & 11.11 & 2.287 \\
\hline \hline
\end{tabular}

display the parameters $a, b, a^{\prime}$, and $b^{\prime}$ for some of these $n$ and $n^{\prime}$.

As in Ref. 15, we also compare the performance of graded and mixed devices $(N=3$, with the middle layer of 50 $\mathrm{nm}$ formed by a blend of NPB and $\mathrm{Alq}_{3}$ ). For a given $n, n^{\prime}$, and $r$ we determine $V / J^{1 / 2}$ for the mixed device assuming $B_{12}=0.98$ and $B_{23}=0.02$ (the values used for the graded case). We define $r_{\min }$ as the blend concentration for which $V / J^{1 / 2}$ is minimum. Results are displayed in Table I, where $\Delta_{P}$ shows how much the power consumption in the mixed device is larger than in the graded one for the same total current. It is clear that the graded devices have better performances. The optimization follows a pattern: $\Delta_{P}$ increases for smaller $n$ and $n^{\prime}$. We also observe that the smallest absolute value of $V / J^{1 / 2}$, both in graded and mixed devices, is obtained for $n=n^{\prime}=2$.

Due to the uncertainty in the values of $\mu$, it is important to investigate how much the minority charge carrier mobilities of pure ETM and HTM influence the concentration profile for maximum efficiency. To do so, we assume $n=n^{\prime}$ $=3 / 2$ and keep all the mobilities as in Eq. (12), except by either $\mu_{p}\left(\mathrm{Alq}_{3}\right)$ or $\mu_{n}(\mathrm{NPB})$, whose values we vary within one order of magnitude. We see from the results in Table III that such mobilities considerably influence the optimum profiles. Thus, good measurement of minority charge mobilites are highly desirable.

From Tables I and III we see that for a given set of mobilities parameters, the concentration profile of the optimized graded device is closely related to the values of $r_{\text {min }}$ that optimize the mixed device performance: for $r_{\min }<0.5$ $(>0.5)$ the concentration profile tends to have a smaller (greater) concentration of NPB. In the case of $r_{\min } \sim 0.5$, the concentration profile tends to be symmetric

TABLE III. Concentration profiles of $\mathrm{Alq}_{3}$ and NPB that optimize the performance of devices with $n=n^{\prime}=3 / 2$ and one of the mobilities in Eq. (12) changed to: (a) $5 \times 10^{-8} \mathrm{~cm}^{2} / \mathrm{V} \mathrm{s}$, (b) $2 \times 10^{-7} \mathrm{~cm}^{2} / \mathrm{V} \mathrm{s}$, (c) $3 \times 10^{-7} \mathrm{~cm}^{2} / \mathrm{V} \mathrm{s}$, and (d) $5 \times 10^{-7} \mathrm{~cm}^{2} / \mathrm{V} \mathrm{s}$.

\begin{tabular}{lcccc}
\hline \hline & & $V / J^{1 / 2}$ & & \\
Changed $\mu$ & {$\left[\mathrm{Alq}_{3}\right]:[\mathrm{NPB}]$} & grad. \{mix. $\}$ & $r_{\text {min }}$ & $\Delta_{P}$ \\
\hline$\mu_{p}\left(\mathrm{Alq}_{3}\right)^{(\mathrm{a})}$ & $1: 1,1: 2,1: 3,1: 5,1: 7$ & $23.8\{25.7\}$ & 0.70 & $7 \%$ \\
$\mu_{n}(\mathrm{NPB})^{(\mathrm{a})}$ & $8: 1,4: 1,2: 1,1: 1,1: 1$ & $23.5\{25.8\}$ & 0.29 & $9 \%$ \\
$\left.\mu_{p}(\mathrm{Alq})_{3}\right)^{(\mathrm{b})}$ & $7: 1,5: 1,2: 1,1: 1,1: 1$ & $16.7\{18.4\}$ & 0.29 & $9 \%$ \\
$\mu_{n}(\mathrm{NPB})^{(\mathrm{b})}$ & $1: 1,1: 1,1: 3,1: 5,1: 7$ & $16.7\{18.3\}$ & 0.70 & $9 \%$ \\
$\left.\mu_{p}(\mathrm{Alq})_{3}\right)^{(\mathrm{c})}$ & $10: 1,7: 1,4: 1,2: 1,1: 1$ & $14.6\{15.9\}$ & 0.19 & $8 \%$ \\
$\mu_{n}(\mathrm{NPB})^{(\mathrm{c})}$ & $1: 1,1: 1,1: 3,1: 5,1: 7$ & $14.5\{15.5\}$ & 0.79 & $6 \%$ \\
$\left.\mu_{p}(\mathrm{Alq})_{3}\right)^{(\mathrm{d})}$ & $15: 1,9: 1,7: 1,4: 1,2: 1$ & $12.1\{12.9\}$ & 0.11 & $6 \%$ \\
$\mu_{n}(\mathrm{NPB})^{(\mathrm{d})}$ & $1: 2,1: 3,1: 6,1: 12,1: 20$ & $11.9\{12.7\}$ & 0.87 & $7 \%$ \\
\hline \hline
\end{tabular}


TABLE IV. $V / J^{1 / 2}$ for various concentration profiles of $\mathrm{Alq}_{3}$ and NPB. The parameters values are the ones of the third row of Table III.

\begin{tabular}{cc}
\hline \hline$\left[\mathrm{Alq}_{3}\right]:[\mathrm{NPB}]$ & $V / J^{1 / 2}$ \\
\hline $7: 1,5: 1,2: 1,1: 1,1: 1$ & 16.7 \\
$9: 1,5: 1,1: 1,1: 2,1: 3$ & 17.4 \\
$5: 1,3: 1,1: 1,1: 2,1: 3$ & 17.5 \\
$9: 1,7: 1,5: 1,3: 1,2: 1$ & 17.8 \\
$2: 1,1: 1,1: 2,1: 3,1: 4$ & 23.0 \\
\hline
\end{tabular}

([NPB]:[ $\left.\left[\mathrm{Alq}_{3}\right] \leftrightarrow\left[\mathrm{Alq}_{3}\right]:[\mathrm{NPB}]\right)$ around the fourth layer. This behavior was observed for a wide range of parameters values.

Our model predicts that $V / J^{1 / 2}$ is relatively stable with respect to small changes in the concentration profile that minimizes it. In Table IV we show $V / J^{1 / 2}$ for five different concentration profiles for the case of $n=n^{\prime}=3 / 2$ and the mobilities given by Eq. (12), except for $\mu_{p}\left(\mathrm{Alq}_{3}\right)$ which we assume to be $2 \times 10^{-7} \mathrm{~cm}^{2} / \mathrm{V} \mathrm{s}$ (third row of Table III). The point is illustrated by comparing the first four rows with the last row of Table IV.

All the present qualitative predictions agree with results obtained for the graded devices studied in Ref. 15. In Fig. 3 we display experimental current-voltage characteristics for one mixed and three graded devices. The three concentration profiles are: graded I: 9:1, 7:1, 5:1, 3:1, 1:1; graded II: 9:1, $7: 1,5: 2,1: 1,1: 3$; and graded III: 5:1, 5:3, 1:1, 3:5, 1:5. In the mixed device, the concentration which leads to the maximum electroluminescent (EL) efficiency is $r_{\min }=0.167$ $\left(\mathrm{Alq}_{3}: \mathrm{NPB}=5: 1\right)$. It is clear from Fig. 3 that $V / J^{1 / 2}$ is smaller for the graded devices. We also observe that $V / J^{1 / 2}$ is almost the same for the three graded devices, which were chosen in order to optimize the device performance (brightness and power consumption). As the three concentration profiles are similar, one may conclude that $V / J^{1 / 2}$ is not too sensitive to changes in the concentration profiles around the optimum value, in agreement with our previous discussion.

\section{DISCUSSION}

We have seen that a graded emissive multilayer implies a 9\%-13\% decrease in power consumption when compared to the best performance of the mixed case. Besides the electric response, the brightness in such devices is also of fundamen-

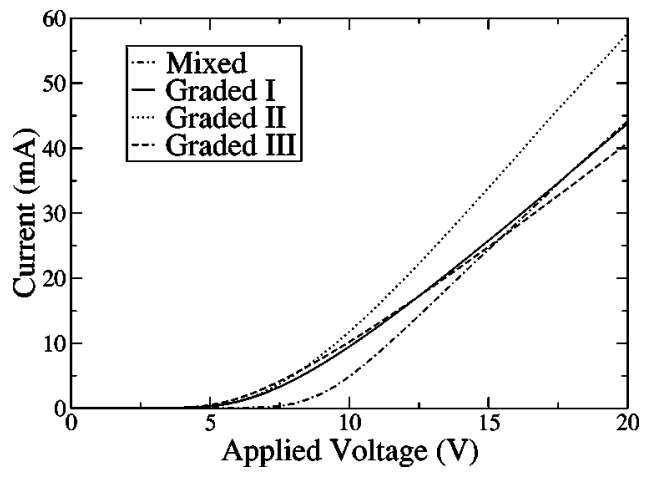

FIG. 3. Current-voltage characteristics for graded and mixed devices of Ref. 15. tal importance. In several cases the brightness $(\mathcal{B})$ of OLEDS is proportional to the current, with the proportionality constant being the EL efficiency $\left(\mathcal{B}=C_{\text {eff }} J\right)$. This relation holds for the graded and mixed devices in Ref. 15 for current densities up to $200 \mathrm{~mA} / \mathrm{cm}^{2}$. An important aspect is that $C_{\text {eff }}$ is a function of $r$. In the graded case we have an EL efficiency for each layer, so that

$$
\mathcal{C}_{\text {eff }}^{\text {graded }}=\sum_{i=2}^{i=N-1} C_{\text {eff }}^{(i)}
$$

It is possible to compare (for the same applied voltage) the brightness of the graded and mixed devices if $C_{\mathrm{eff}}^{\text {graded }}$ and $C_{\text {eff }}^{\text {mixed }}$ are known. Our model predicts an interesting relation between $r_{\min }$ for mixed devices and the optimized concentration profile for graded devices: the $r$ value for the middle layer as well as the average $r$ for all layers is approximately equal to $r_{\min }$. As a consequence, if $C_{\text {eff }}$ has an approximately linear dependence on $r$, we can assume $C_{\text {eff }}^{\text {mixed }} \sim C_{\text {eff }}^{\text {graded }}$. In this case, we find that the brightness of the graded device will be $20 \%-30 \%$ higher than that of the mixed one. This is compatible with the results for the brightness seen in Fig. 3 and Table I of Ref. 15.

As explained before, we do not include the pure HTM and ETM layers in our simulations in order to avoid problems due to the numerical sensitivity to the boundary conditions. Even with this simplification, we were able to describe the different features observed in real graded and mixed OLEDs. We have assumed that the fraction of negative carrier current at the interfaces between the external layers and the mixed region were the same as those in the graded case. Nevertheless, we observe that they hardly have the same values. With the present model, the inclusion of the pure layers would cause some difficulty in fitting the experimental data (since there are always errors in determining $B_{a}$ and $B_{c}$ ). However, for a qualitative analysis we can include them and set the same particular values of $B_{a}$ and $B_{c}$ in both the graded and mixed devices. We find that, for reasonable values of $B_{c}$ and $B_{a}$, in the mixed case $B_{12}\left(B_{23}\right)$ is always smaller (larger) than $0.98(0.02)$, the values used in the graded case for $B_{12}$ and $B_{N-1 N}$. Larger $B_{23}$ implies a lower electron-hole recombination taking place at the mixed layer. Since we assume that the total emitted light is proportional to the amount of electron-hole recombination in the emissive layers, we can expect a decrease in the total emitted light for the mixed device (from $3 \%$ to 5\%), so strengthening our previous conclusion.

Some self-consistency checks can be made. The first is the assumption about the electric field independence of the mobilities and its consequence in analyzing the experimental data. In Fig. 4 we show a typical electric field calculated inside the graded region. For such a variation along the device, the mobilities for NPB and $\mathrm{Alq}_{3}$ remain basically unchanged according to the recent measurements of Refs. 25 and 26. Some change could be expected for the hole mobility in $\mathrm{Alq}_{3}$ which, however, would not drastically alter our optimization results. The second is the assumption that most of the electron-hole recombination occurs inside the graded region. To verify this, in Fig. 5 we show the recombination 


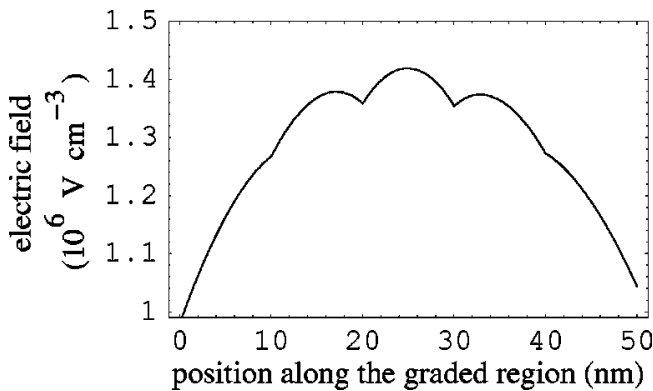

FIG. 4. Typical electric field profile in the graded region with five layers. Here $J=0.1 \mathrm{~A} \mathrm{~cm}^{-2}$.

rate, $\gamma N P$, along the five inner layers for the same parameters as in Fig. 4. It is clear that most of the recombination takes place at the central part of this region, where the values of two carrier mobilities are smaller and closer to each other. This fact represents another important advantage of graded devices: in single layer devices most of the electron-hole pairs are formed near the cathode, whereas light emission is suppressed in this region due to quenching losses at the metal layer, strongly limiting device performance. In graded devices the recombination events are expected to occur mostly in the central region between cathode and anode. Due to our assumptions, the curve of Fig. 5 essentially represents the electron-hole pairs density, indicating that electron-hole pair formation near electrodes is significantly less important than in homogeneous layer devices, making these devices less susceptible to electrode quenching losses.

Finally, the third check is the model prediction of a soft variation of $r$ along the layers of the graded region at the optimum condition. For instance, for the case shown in the third row of Table I we have $r=0.25,0.33,0.5,0.67,0.75$. Such a result corroborates the assumption of continuity of both the electric displacement and $B$ at the interfaces in the graded region. The variations of the mobilities from pure ETM and pure HTM to their first neighbor graded layers do not represent a problem, since at those interfaces we have essentially only one charge carrier and everything behaves like in Fig. 4 of Ref. 20, i.e., with almost no charge accumulation. We show in Fig. 6 a typical example of electron charge density and $\mu_{n} \times$ electron charge density as functions of the position in the graded region. Since the electric field is continuous at the interfaces, the discontinuities of the volumetric charge density are then a consequence of the different

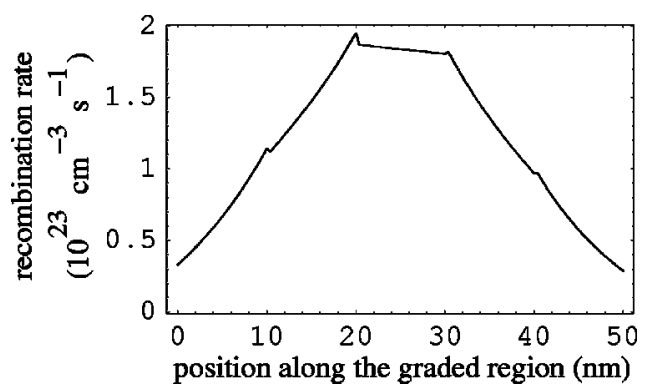

FIG. 5. Recombination profile for the parameters as in Fig. 4.

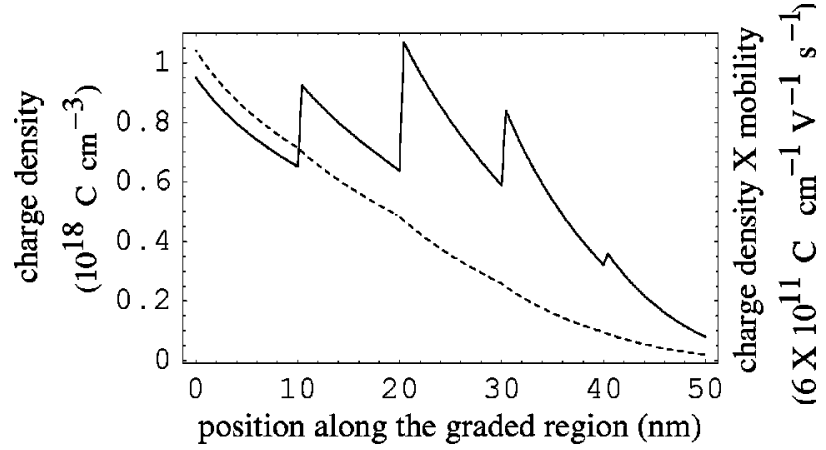

FIG. 6. Electron charge density (full line) and electron charge density $\times$ effective electron mobility (broken line) for the same situation as in Fig. 4.

mobilities. The positive charge density shows a similar behavior.

We have considered the graded region with five layers, since it allows a better comparison between our predictions and the experimental data for the graded device of Ref. 15. However, we also have investigated the case of graded regions (of $50 \mathrm{~nm}$ ) formed by four and six layers of equal thickness. The simulations show that by controlling the concentration profiles, it is possible to reach essentially the same $V / J^{1 / 2}$ minima.

\section{CONCLUSION}

In this contribution we developed a simple model to analyze the electric properties of graded multilayer OLEDs. It is an analytical method where all the expressions are given in closed form unless for some calculations like inverting equations as Eq. (5) or obtaining matching conditions as in Eq. (8), which are done quite easily using numerical methods. The model can be used to find (through simulations) the parameters that optimize device efficiency. The only requirement is that one needs to know how the electron and hole effective mobilites depend on the blend concentration. In particular, we have discussed the case where those mobilities behave as powers of $r$, and do not depend too strongly on the electric field. The predictions of the model were tested by comparing them with experimental data for NPB and $\mathrm{Alq}_{3}$ and we found very good agreement.

Although the present model is already an important step towards the understanding of graded OLEDs, further theoretical and experimental investigation are necessary.

\section{ACKNOWLEDGMENTS}

A.G. would like to thank the Physics Department at UFPR for their kind hospitality during a stay in which part of this work was carried out. FAPESP-Brazil (A.G.), CNPqBrazil (I.A.H and M.G.E.L) and "Hundreds Talents Program" of Chinese Academy of Science (D.M.) are gratefully acknowledged for research fellowships.

${ }^{1}$ W. Helfrich and W. G. Schneider, Phys. Rev. Lett. 14, 229 (1965).

${ }^{2}$ C. W. Tang and S. A. VanSlyke, Appl. Phys. Lett. 51, 913 (1987).

${ }^{3}$ D. Ma, I. A. Hümmelgen, X. Jing, Z. Hong, L. Wang, X. Zhao, F. Wang, and F. Karasz, J. Appl. Phys. 87, 312 (2000). 
${ }^{4}$ D. Ma, I. A. Hümmelgen, X. Jing, D. Wang, Z. Hong, L. Wang, X. Zhao, and F. Wang, Braz. J. Phys. 30, 392 (2000).

${ }^{5}$ A. G. Werner, F. Li, K. Harada, M. Pfeiffer, T. Fritz, and K. Leo, Appl. Phys. Lett. 82, 4495 (2003).

${ }^{6}$ H. P. Schob and I. Zschokke-Grnacher, Mol. Cryst. Liq. Cryst. 13, 115 (1971).

${ }^{7}$ G. G. Malliaras and J. C. Scott, J. Appl. Phys. 83, 5399 (1998).

${ }^{8}$ Y. Kawabe, M. M. M. Morell, G. E. Jabour, S. E. Shaheen, B. Kippelen, and N Peyghambarian, J. Appl. Phys. 84, 5306 (1998).

${ }^{9}$ M. Koehler, M. G. E. da Luz, and I. A. Hümmelgen, J. Phys. D 33, 2096 (2000).

${ }^{10}$ J. Grüner, M. Remmers, and D. Neher, Adv. Mater. (Weinheim, Ger.) 9, 964 (1997).

${ }^{11}$ N. C. Greenham, S. C. Moratti, D. D. C. Bradley, R. H. Friend, and A. B. Holmes, Nature (London) 365, 628 (1993).

${ }^{12}$ Y. Cao, I. D. Parker, C. Zhang, and A. J. Heeger, Nature (London) 397, 414 (1999).

${ }^{13}$ Y. D. Jing, J. P. Yang, P. L. Heremans, M. Van de Auweraer, E. Rousseau, N. J. Geise, and G. Borghs, Chem. Phys. Lett. 320, 387 (2000).

${ }^{14}$ M. Matsumura and K. Manabe, Appl. Phys. Lett. 79, 4491 (2001).

${ }^{15}$ D. Ma, C. S. Lee, S. T. Lee, and L. S. Hung, Appl. Phys. Lett. 80, 3641 (2002).
${ }^{16}$ R. H. Parmenter and W. Ruppel, J. Appl. Phys. 30, 1548 (1959).

${ }^{17}$ J. C. Scott, S. Karg, and S. A. Carter, J. Appl. Phys. 82, 1454 (1997).

${ }^{18}$ I. S. Gradshteyn and I. M. Ryzhik, Table of Integrals, Series, and Products, 2nd ed. (Academic, San Diego, 1994).

${ }^{19}$ S. Berleb, W. Brütting, and G. Paasch, Org. Elect. 1, 41 (2000).

${ }^{20}$ B. K. Crone, P. S. Davids, I. H. Campbell, and D. L. Smith, J. Appl. Phys. 87, 1974 (2000).

${ }^{21}$ B. Ruhstaller, S. A. Carter, S. Barth, H. Riel, W. Riess, and J. C. Scott, J. Appl. Phys. 89, 4575 (2001).

${ }^{22}$ The concentration of two mixed materials is defined by the ratio $r$ $=[\mathrm{HTM}] /([\mathrm{HTM}]+[\mathrm{ETM}])$, where $[X]$ is either the mass or the number of dopant atoms plus the number of molecules of the doped $X$ material. $r$ also can be obtained from the speed (given in terms of layers per second) at which the HTM and ETM are codeposited. Obviously, $0 \leqslant r \leqslant 1$.

${ }^{23}$ J. A. Reedijk et al., Synth. Met. 101, 475 (1999); Y. Harima, Y. Kunugi, K. Yamashita, and M. Shiotani, Chem. Phys. Lett. 317, 310 (2000).

${ }^{24} \mathrm{D}$. Ma and I. A. Hümmelgen (unpublished).

${ }^{25}$ S. Barth et al., J. Appl. Phys. 89, 3711 (2001); A. G. Mückl, S. Berleb, W. Brütting, and M. Schwoerer, Synth. Met. 111-112, 91 (2000).

${ }^{26}$ J. Kovac et al., Mater. Sci. Eng., B B85, 172 (2001). 